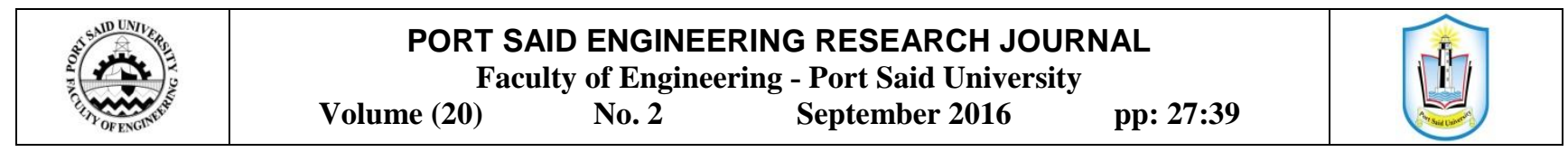

\title{
Analyzing Barrettes as Large-Section Supports by CCT
}

\author{
Hassan Ibrahiml/ Ibrahim El Arabi²/Mahmoud El Gendy ${ }^{3}$
}

\begin{abstract}
Most of soil structure interaction methods for analyzing large-section supports such as barrette foundation modeling the barrette and surrounding soil using 3D FE model. In which, the model leads to a large finite element mesh of a large system of linear equations to be solved. In this paper, a Composed Coefficient Technique (CCT) is adapted for analyzing barrette. The technique takes into account the 3D full interactions between barrette and the surrounding soil. Due to the high rigidity of the barrette relative to the surrounding soil, a uniform or variable settlement along the barrette height can be considered. This enables to compose the stiffness coefficients of the soil matrix into composed coefficients, which consequently leads to a significant reduction in the soil stiffness matrix. An application for analyzing barrette by CCT is carried on the soil of the new area of East Port Said, in where the typical soil stratification is very week and structures in this area need to be supported by deep foundations such as barrettes. The application presents guidelines and diagrams for barrettes that may be used in East Port Said.
\end{abstract}

Key words: Soil structure interaction, Deep foundation, Barrette, Settlement

\section{INTRODUCTION}

Heavy loaded structures need to be supported on deep foundations such as barrette. Analyzing this system of foundation is a complex task because it is a threedimensional problem including the interaction between barrettes and soil. Considering this interaction requires a long computational time where a huge soil matrix is required to verify the compatibility among barrette and soil. The standard models for analyzing this complex problem depend on a full three-dimensional analysis, which leads to very large number of elements, and thus these models are time consuming even for the fast computers of today, especially when analyzing barrette group or barrette raft.

A similar foundation element of pile maybe considered as a less complicated problem than that of the barrette cross section. Piles in most cases are circular in shape with small cross-section area, while that of the barrette is large with a rectangular shape. Therefore, pile can be treated as a beam member exposed to point loads on its nodes, while barrette must be treated as a block member having a cross-section of two dimensions and exposed to uniform loads on its sides.

There are a lot of available methods that are being used to analyze piles most of them are used also to analyze barrette with equivalent cross section area to that of the pile. Some of them are those of Basu et al.[1], Lei et al.[17], and Seo et al.[27]. Other alternative methods to analyze the barrettes are those using the full three dimensional finite element method as that of Fellenius et al. [8].

\footnotetext{
Professor, Faculty of Engineering, Port Said University, Egypt, Email: hi_hgh@yahoo.com

${ }^{2}$ Associate professor, Faculty of Engineering, Port Said University, Egypt, Email: iielarabiii@gmail.com

3 Graduate student, Faculty of Engineering, Port Said University Egypt, Email: mahmoud_gendy1@hotmail.com
}

Some other methods depend on load settlement tests of barrette either on the field or in the lab. Some of them are those of Thasnanipan et al.[29], Thasnanipan et al.[30], Shulyatiev et al.[28], and Lin et al.[18]. For single pile, pile group and piled raft, El Gendy [4] presented a technique based on the flexibility coefficients an efficient analysis by using Composed Coefficient Technique (CCT) to reduce the size of the entire soil stiffness matrix. In this technique, the pile is treated as a rigid member having a uniform settlement for all nodes along its shaft and base. $C C T$ enables to assemble pile coefficients in composed coefficients. This technique is applied efficiently for many studies, some of them are those of Hattab [10], Reda [25], Rabiei [22], [23], [24], Kamash, W. [12] [13] [14] [15], Kamash et al. [16], Ibrahim et al. [11], Mobarak W. [19], El-Labban [7], Moubarak A. [20], Chieruzzi et al. [3], and El Gendy et al. [5], [6].

This technique is also further developed to be used in this study for analyzing the barrette based on both flexibility coefficients and full 3D FE. The Advantage of the $C C T$ is that interaction of soil elements with the barrette elements are taken into consideration. The proposed analysis reduces considerably the number of equations that needs to be solved. Another point of view to choice of the $C C T$ for the barrette analysis is that the designer is interested in the soil settlements and contact forces at different levels on the barrette height not at each barrette node. Another problem can be tread by $C C T$ is the nonlinearity of the barrette load-settlement behavior.

\section{MATHEMATICAL MODELING}

\subsection{Modeling Single Barrette Using Flexibility Coefficients}


Following the $C C T$ for modeling pile foundation by $E l$ Gendy [4], a composed coefficient $k_{s} \quad[\mathrm{kN} / \mathrm{m}]$ representing the soil stiffness of the barrette is developed. The mathematical formulation of the composed coefficient $k_{s}$ for different cases of barrette analyses will be described in the forthcoming items.

\subsubsection{Soil Stiffness Matrix}

The rectangular cross sectional barrette shown in Figure 1 is divided into a number of shaft elements and base elements with $n_{s}$ nodes, each acted upon by a distributed stress. To carry out the analysis, the stresses acting on shaft and base elements are replaced by a series of concentrated forces acting on nodes.

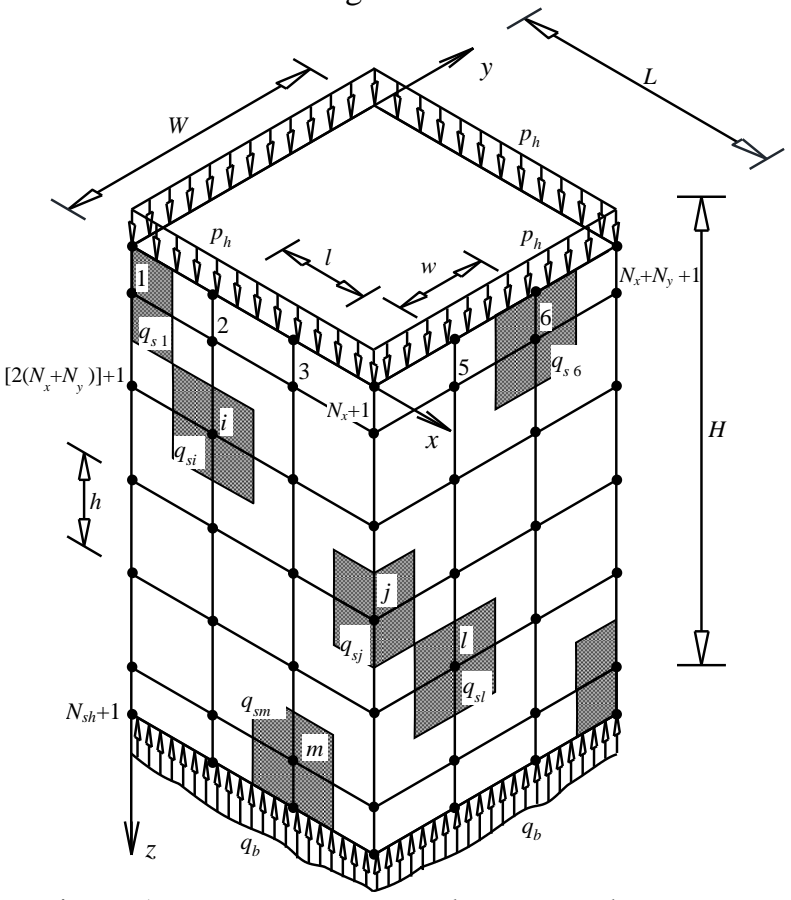

Figure 1 Barrette geometry, elements and stresses.

The settlement of the soil at any node $i$ of the barrette may be rewritten in general form as:

$$
s_{i}=\sum_{j=1}^{n_{s}} I_{i, j} Q_{j}
$$

where: $s_{i}$ Soil settlement on any node $i$ either on the shaft or on the base, [m]; $Q_{j}$ Contact force on node $j,[\mathrm{kN}] ; Q_{j}$ represents either the forces on shaft nodes or base nodes; $n_{s}$ Total number of contact nodes; $I_{i}, j$ Flexibility coefficient of node $i$ due to a unit force on node $j$ $[\mathrm{m} / \mathrm{kN}]$. Closed form equations for these coefficients are described in the Appendix A.

Eq. (1) for settlements of the soil adjacent to all nodes of the barrette may be written in a matrix form as:

$$
\{s\}=[I s]\{Q\}
$$

Where $\{s\}$ is $n_{s}$ settlement vector; $\{Q\}$ is $n_{s}$ contact force vector; $[I s]$ is $n_{s} \times n_{s}$ soil flexibility matrix.

Inverting the soil flexibility matrix in Eq. (2), leads to:

$$
\{Q\}=[k s]\{s\}
$$

Where $[k s]$ is $n_{s} \times n_{s}$ soil stiffness matrix, $[k s]=[I s]^{-1}$.

\subsubsection{Rigid Analysis}

The barrette is a huge concrete volume which may be considered as a rigid body subjected to vertical loads and moves vertically with a uniform displacement $w o=s_{I}$ $=s_{2}=\ldots=s_{n s}$ on all its nodes. Therefore, the unknowns of the problem are reduced to $n_{s}$ contact forces and the rigid body displacement wo. Carrying out the summation of all contact forces in the Eq. (3), leads to:

$$
\sum_{i=1}^{n_{s}} Q_{i}=w o \sum_{i=1}^{n_{s}} \sum_{j=1}^{n_{s}} k_{i, j}
$$

Where $k_{i, j}$ are the coefficients of the soil stiffness matrix $[k s]$.

Equation (4) may be rewritten as:

$$
P h=k s w o
$$

Where the applied force $P h[\mathrm{kN}]$ on the barrette head is the sum of all contact forces $Q_{i}, P h=\sum_{i=1}^{n_{s}} Q_{i}$, while the composed coefficient $k s[\mathrm{kN} / \mathrm{m}]$ is the sum of all coefficients of the soil stiffness matrix, $k s=\sum_{i=1}^{n_{s}} \sum_{j=1}^{n_{s}} k_{i, j}$.

Equation (5) gives the linear relation between the applied load on the barrette head and the uniform settlement wo of all barrette nodes. For a single barrette, the applied load on the barrette head $P h$ is given and hence the uniform settlement wo can be determined from Eq. (5). Substituting the value of $w o$ in Eq. (3), gives $n_{\mathrm{s}}$ unknown contact forces $Q_{i}$ in case of considering the barrette as full rigid body.

\subsubsection{Elastic Analysis}

In this analysis, the elasticity of the barrette is considered. The finite element method is used for analyzing the barrette body, which is exposed to external forces on soil-barrette interface as soil reactions in addition to the applied load on its head as an action. A compatibility between the vertical displacements of the barrette and the soil settlements at the soil-barrette interface is taken in the vertical direction only. This assumption is related to that the external load on the barrette head, which is expected to be heavy load, is applied in the vertical direction. For comparative examinations and to avoid a huge barrette stiffness matrix, the barrette elasticity is determined from the finite element method using either $1 \mathrm{D}$ or $3 \mathrm{D}$ finite elements.

\subsubsection{Elastic Analysis Using 1D Finite Elements}

To simplify the solution of the analysis and to reduce the size of the problem, the barrette is represented by a vertical line member having a variable settlement (or vertical displacement) along its height. Another point of view in choosing this idea is that the designer is interested in the soil settlements and contact forces at different levels on the barrette height not at each barrette node. In the proposed analysis, the composed coefficient technique is used to perform a soil stiffness matrix for barrette as a line member from the original soil stiffness matrix of Eq. (3). This soil stiffness matrix takes also into account the interaction effect among all soil-barrette interface nodes. 


\subsection{Soil Stiffness Matrix}

To describe the formulation of composed coefficients for generating the soil stiffness matrix of the barrette in this case, consider, as an example, the simple barrette shown in Figure 2a, which has a total of $n=33$ surface nodes. The barrette of $3 \mathrm{D}$ is converted to $1 \mathrm{D}$ as indicated in Figure $2 \mathrm{~b}$, which has $n_{b}=4$ nodes in 4 levels only. Each node has a force and a settlement in the vertical direction. The unknowns of the problem will be reduced to $n_{b}$ contact forces $Q_{b i}$ on soil-barrette interface and $n_{b}$ settlements (or displacements) $s_{b i}$ on all nodes of the barrette in the vertical direction.
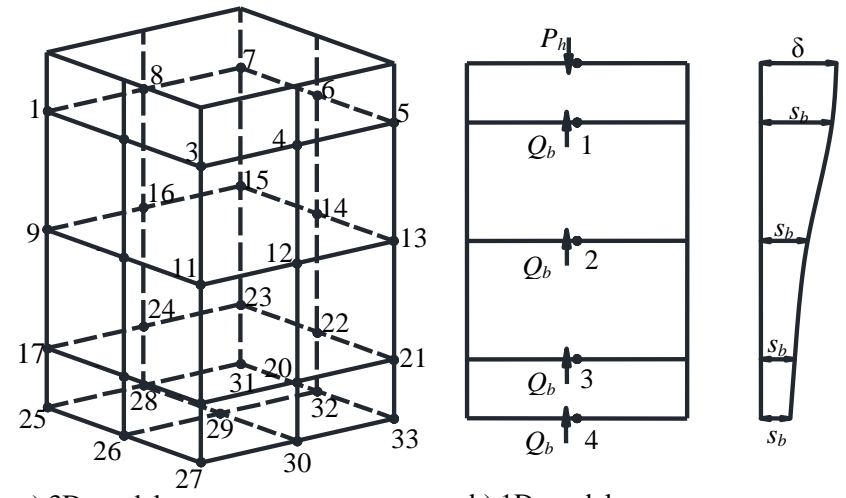

a) 3D-mode

b) 1D-model

Figure 2 Surface mesh of the barrette with node numbering, loads and settlements.

The soil stiffness matrix of Eq. (3) for the barrette shown in Figure 2a can be expanded in the following matrix equation:

$$
\left\{\begin{array}{l}
\left\{\begin{array}{l}
Q_{1} \\
\ldots \\
Q_{8}
\end{array}\right\}_{1} \\
\ldots \\
\left.\left\{\begin{array}{l}
Q_{25} \\
\ldots \\
Q_{33}
\end{array}\right\}_{4}\right\}=\left[\begin{array}{ccccccc}
k_{1,1} & \ldots & k_{1,8} & \ldots & k_{1,25} & \ldots & k_{1,33} \\
\ldots & \ldots & \ldots & \ldots & \ldots & \ldots & \ldots \\
k_{8,1} & \ldots & k_{8,8} & \ldots & k_{8,25} & \ldots & k_{8,33} \\
\ldots & \ldots & \ldots & \ldots & \ldots & \ldots & \ldots \\
k_{25,1} & \ldots & k_{25,8} & \ldots & k_{25,25} & \ldots & k_{25,33} \\
\ldots & \ldots & \ldots & \ldots & \ldots & \ldots & \ldots \\
k_{33,1} & \ldots & k_{33,8} & \ldots & k_{33,25} & \ldots & k_{33,33}
\end{array}\right]\left\{\begin{array}{l}
s_{1} \\
\ldots \\
s_{8} \\
\ldots \\
\ldots \\
s_{25} \\
\ldots \\
s_{33}
\end{array}\right\} \\
4
\end{array}\right\}
$$

In Eq. (6), carrying out the summation of rows and columns corresponding to the barrette node $i$ in $1 \mathrm{D}$, leads to:

$$
\left\{\begin{array}{l}
\left\{\sum_{i=1}^{8} Q_{i}\right\}_{1} \\
\cdots \\
\left\{\sum_{i=25}^{33} Q_{i}\right\}_{4}
\end{array}\right\}=\left[\begin{array}{ccc}
\sum_{i=1}^{8} \sum_{j=1}^{8} k_{i, j} & \ldots & \sum_{i=1}^{8} \sum_{j=25}^{32} k_{i, j} \\
\ldots & \ldots & \ldots \\
\sum_{i=25}^{33} \sum_{j=1}^{8} k_{i, j} & \cdots & \sum_{i=25}^{33} \sum_{j=25}^{33} k_{i, j}
\end{array}\right]\left\{\begin{array}{l}
s_{b 1} \\
\cdots \\
s_{b 4}
\end{array}\right\}
$$

Accordingly, Eq. (7) of soil stiffness matrix can be rewritten for the barrette of $1 \mathrm{D}$ in composed coefficients as:

$$
\left\{\begin{array}{l}
Q_{b 1} \\
Q_{b 2} \\
Q_{b 3} \\
Q_{b 4}
\end{array}\right\}=\left[\begin{array}{llll}
K_{1,1} & K_{1,2} & K_{1,3} & K_{1,4} \\
K_{2,1} & K_{2,2} & K_{2,3} & K_{2,4} \\
K_{3,1} & K_{3,2} & K_{3,3} & K_{2,4} \\
K_{4,1} & K_{4,2} & K_{4,3} & K_{2,4}
\end{array}\right]\left\{\begin{array}{c}
s_{b 1} \\
s_{b 2} \\
s_{b 3} \\
s_{b 4}
\end{array}\right\}
$$

where $K_{i, j}$ is composed coefficient, $[\mathrm{kN} / \mathrm{m}] ; s_{b i}$ is settlement in node $i$ of $1 \mathrm{D}$ barrette, [m], $s_{b 1}=s_{1}=s_{2}=\ldots=$ $s_{8}, s_{b 2}=s_{9}=s_{10}=\ldots=s_{16}, \ldots \ldots, s_{b 4}=s_{25}=s_{26}=\ldots=s_{33} ; Q_{b i}$ is contact force on node $i$ of $1 \mathrm{D}$ barrette [kN], $Q_{b 1}=Q_{1+} Q_{2+}$ $\ldots+Q_{8}, Q_{b 2}=Q_{9+} Q_{10+} \ldots+Q_{16, \ldots \ldots,} Q_{b 4=} Q_{25+} Q_{26+} \ldots+Q_{33}$
Eq. (8) shows that the soil stiffness matrix in Eq. (6) of size $33 \times 33$ is reduced considerably to an equivalent soil stiffness matrix of size $4 \times 4$. It could be written in a compacted form as:

$$
\left\{Q_{b}\right\}=[k b]\left\{s_{b}\right\}
$$

\subsection{Barrette Stiffness Matrix}

Using finite element method in the analysis of barrette, only the axial compression of the barrette is considered in determining displacements of barrette elements. The beam stiffness matrix of the barrette element $i$ can be expressed as (Figure 3):

$$
[k p]_{i}=\frac{E p \cdot A p_{i}}{l_{i}}\left[\begin{array}{cc}
1 & -1 \\
-1 & 1
\end{array}\right]
$$

Where $E p$ is Modulus of Elasticity of the barrette material, $\left[\mathrm{kN} / \mathrm{m}^{2}\right] ; A p_{i}$ is cross-section area of the barrette element $i,\left[\mathrm{~m}^{2}\right] ; l_{i}$ is length of the barrette element $i,[\mathrm{~m}]$.

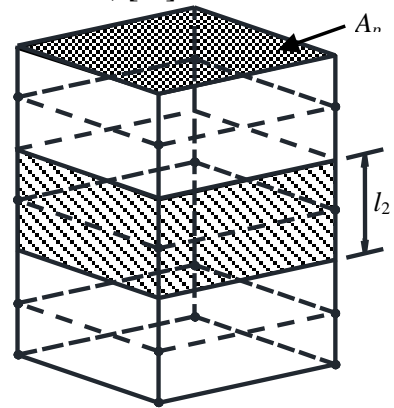

a) Barrette model in 1D-FE

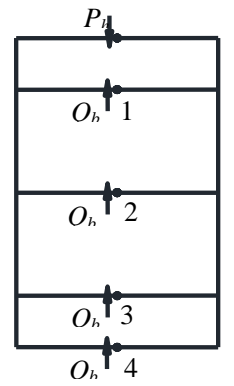

b) Forces and settlements
Figure 3 Finite element mesh of barrette and element geometry.

According to the principal of the finite element method, the assembled axial stiffness matrix equation for the barrette can be written as:

$$
[k p]\{\delta\}=\{P\}-\left\{Q_{b}\right\}
$$

Where $\{\delta\}$ is $\left(n_{\mathrm{s}}+1\right)$ Displacement vector; $\{P\}$ is $\left(n_{\mathrm{s}}+1\right)$ vector of applied load on the barrette, $\{P\}=\{P h, 0,0$, $0, \ldots, 0\}^{\mathrm{T}} ;[k p]$ is $\left(n_{\mathrm{s}}+1\right) \times\left(n_{\mathrm{s}}+1\right)$ beam stiffness matrix.

Substituting Eq. (9) into Eq (11), leads to:

$$
[k p]\{\delta\}=\{P\}-[k b]\left\{s_{b}\right\}
$$

Assuming full compatibility between barrette displacement $\delta_{i}$ and soil settlement $s_{b i}$, the following equation can be obtained:

$$
\llbracket k p]+[k b]\{\delta\}=\{P\}
$$

Solving the above system of linear equations, gives the displacement at each node, which is equal to the soil settlement at that node. Substituting soil settlements from Eq. (13) into Eq. (9), gives contact forces $Q_{b i}$ on the barrette.

\subsubsection{Elastic Analysis Using 3D Finite Elements}

In this case, there is no approximation has to be carried out when determining the elasticity of the barrette itself due to its material, where the barrette is divided into Hexahedra solid elements. Figure 4 shows the mesh of the 3D finite elements of the barrette with loads. Each 
element consist of eight nodes, each node has three forces and three displacements in the three directions. More details concerning this type of the solid element may be found in Chandrupatla and Belegundu [2]. The unknowns of the problem are $n_{s}$ contact forces on soilbarrette interface and $n_{t}$ displacements (or settlements) on all nodes of the barrette in the three directions.
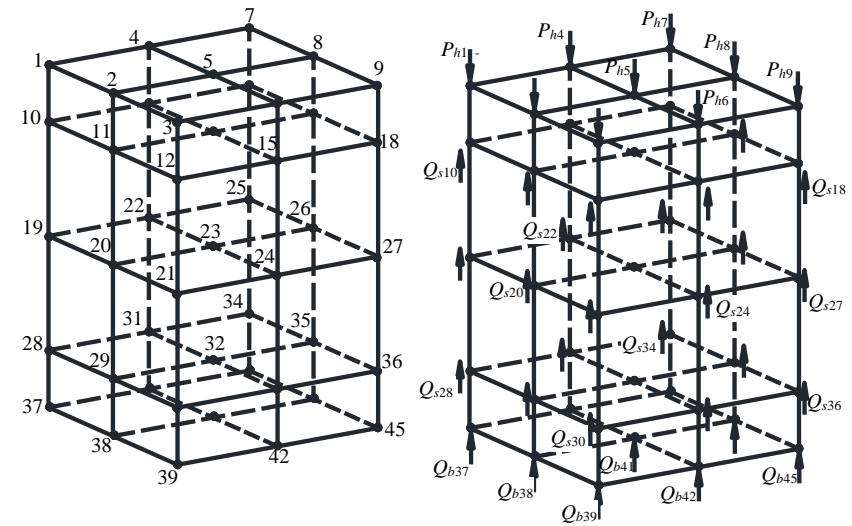

Figure 4 Mesh of the barrette with node numbering, loads and settlements.

According to the finite element method principals, the stiffness matrix equation for the barrette can be defined as:

$$
[k p]\{\delta\}=\{P\}-\{Q\}
$$

Where $\{\delta\}$ is $n_{t}$ displacement vector of displacements $w_{i}$, $u_{i}$ and $v_{i}$ in $z-, x$ - and $y$-directions respectively; $\{P\}$ is $n_{h}$ vector of applied forces on the barrette head; $\{Q\}$ is $n_{s}$ vector of contact forces on the soil-barrette interface; [kp] is $\left(n_{t} \times n_{t}\right)$ barrette stiffness matrix; $n_{h}$ is number of nodes on the barrette head; $n_{s}$ is number of nodes on the soil-barrette interface; $n_{t}$ is total number of barrette nodes, $n_{t=} n_{h+} n_{s}$.

Substituting Eq. (9) into Eq (14), leads to:

$$
[k p]\{\delta\}=\{P\}-[k s]\{s\}
$$

The soil stiffness matrix $[k s]$ is a full matrix, while the original size of the barrette stiffness matrix $[k p]$ is a banded matrix. Therefore, the matrix $[k p]$ is extended to be a full matrix of size $n_{t} * n_{t}$ to enable the summation process of the barrette stiffness matrix with soil stiffness matrix to be carried out.

Assuming full compatibility between barrette displacements $w_{i}$ and the surrounding soil settlement $s_{i}$, the following equation can be obtained:

$$
[[k p]+[k s]\{\delta\}=\{P\}
$$

Solving the above system of linear equations, gives the vertical displacements at each node $w_{i}$, which equal to the soil settlement $s_{i}$ at that node. Substituting soil settlements from Eq. (16) into Eq. (9), gives contact forces $Q_{i}$ on the barrette in case of considering the barrette as an elastic body.

\subsection{Modeling Barrette and Subsoil Using 3D Finite Elements}

The barrette and the surrounding subsoil are represented by $3 \mathrm{D}$ finite elements as shown in Figure 5, which presented a quarter of the mesh and barrettes. Then, the finite element method is used for analyzing the barrette and subsoil medium together using solid block elements. Each element consists of eight nodes, each node has forces and displacements in the three directions. The composed coefficient technique is used to perform the analysis of the single barrette and barrette group.

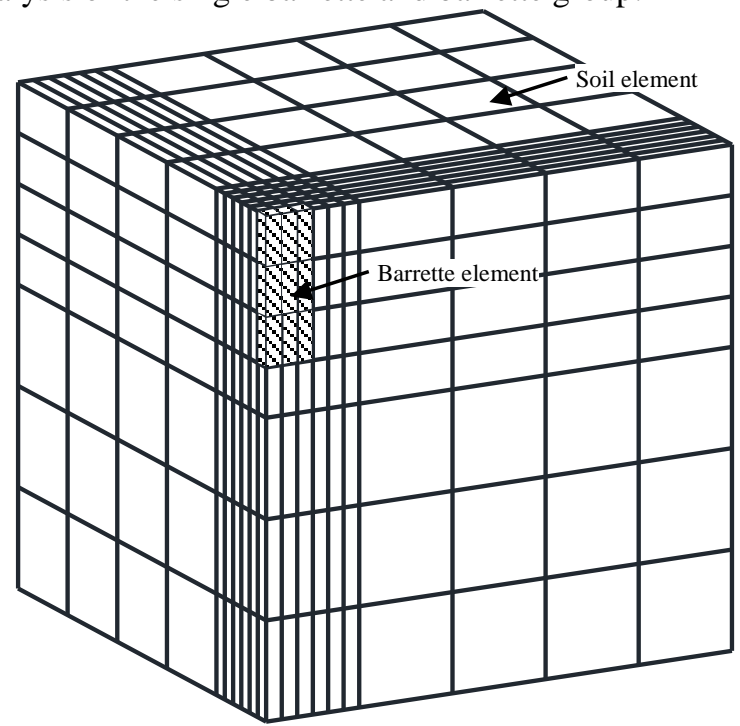

Figure 5 Quarter mesh of 3D finite elements of a barrette and the surrounding subsoil.

The next paragraphs illustrate the generation of a stiffness matrix of composed coefficients for a single barrette. The same procedure can be applied for barrette groups or barrette raft. Consider the simple finite element mesh in the cross section of a barrette and subsoil shown in Figure 6 as an example. The subsoil has the nodes from 1 to 69 , while the barrette has the nodes 70 to 90 .

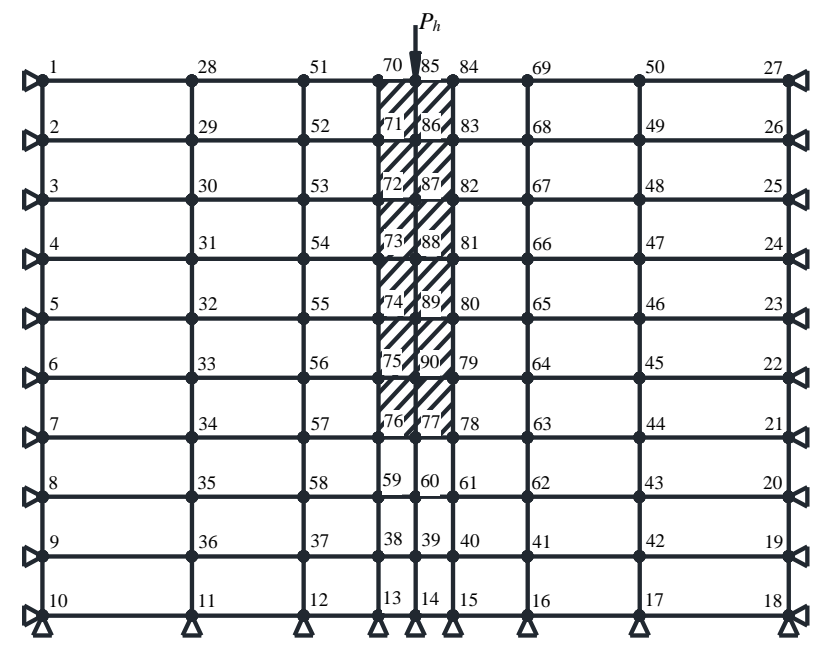

Figure 6 Simple finite element mesh in a cross section of the barrette and subsoil.

The global stiffness matrix equation of the system of the single barrette and the surrounding subsoil can be expressed as:

$$
\{P\}=[k p]\{\delta\}
$$

Where $\{\delta\}$ is $3 n$ displacement vector $\{u, v, w\} ;\{P\}$ is $3 n$ vector of applied load $\left\{p_{x}, p_{y}, p_{z}\right\} ;[k p]$ is $(3 n \times 3 n)$ Stiffness matrix; $n$ is number of the total nodes. 
The barrette nodes in the matrix equation, Eq. (17) are rearranged to be at the end of the matrix. Considering uniform displacements in the three directions due to the high barrette rigidity and carrying out the summation of the corresponding stiffness coefficients, Eq. (17) can be rewritten and expanded as:

$$
\left\{\begin{array}{l}
p_{x 1} \\
p_{y 1} \\
p_{z 1} \\
\ldots \\
p_{x 69} \\
p_{y 69} \\
p_{z 69} \\
p_{x 70} \\
p_{y 70} \\
p_{z 70} \\
\ldots \\
p_{x 90} \\
p_{y 90} \\
p_{z 90}
\end{array}\right\}=\left[\begin{array}{cccccccccccccc}
k_{1,1} & k_{1,2} & k_{1,3} & \ldots & k_{1,205} & \ldots & \ldots & \ldots & \ldots & k_{1,210} & \ldots & k_{1,268} & k_{1,269} & k_{1,270} \\
k_{2,1} & k_{2,2} & k_{2,3} & \ldots & k_{2,205} & \ldots & \ldots & \ldots & \ldots & k_{2,210} & \ldots & k_{2,268} & k_{2,269} & k_{2,270} \\
k_{3,1} & k_{3,2} & k_{3,3} & \ldots & k_{3,205} & \ldots & \ldots & \ldots & \ldots & k_{3,210} & \ldots & k_{3,268} & k_{3,269} & k_{3,270} \\
\ldots & \ldots & \ldots & \ldots & \ldots & \ldots & \ldots & \ldots & \ldots & \ldots & \ldots & \ldots & \ldots & \ldots \\
k_{205,1} & k_{205,2} & k_{205,3} & \ldots & k_{205,205} & \ldots & \ldots & \ldots & \ldots & k_{205,210} & \ldots & k_{205,268} & k_{205,269} & k_{205,270} \\
k_{206,1} & k_{206,2} & k_{2063} & \ldots & k_{206,205} & \ldots & \ldots & \ldots & \ldots & k_{206,210} & \ldots & k_{206,268} & k_{206,269} & k_{206,270} \\
k_{207,1} & k_{207,2} & k_{207,3} & \ldots & k_{207,205} & \ldots & \ldots & \ldots & \ldots & k_{207,210} & \ldots & k_{207,268} & k_{207,269} & k_{207,270} \\
k_{208,1} & k_{208,2} & k_{208,3} & \ldots & k_{208,205} & \ldots & \ldots & \ldots & \ldots & k_{208,210} & \ldots & k_{208,268} & k_{208,269} & k_{208,270} \\
k_{209,1} & k_{209,2} & k_{209,3} & \ldots & k_{209,205} & \ldots & \ldots & \ldots & \ldots & k_{209,210} & \ldots & k_{209,268} & k_{209,269} & k_{209,270} \\
k_{210,1} & k_{210,2} & k_{2103} & \ldots & k_{210,205} & \ldots & \ldots & \ldots & \ldots & k_{210,210} & \ldots & k_{210,268} & k_{210,269} & k_{210,270} \\
\ldots & \ldots & \ldots & \ldots & \ldots & \ldots & \ldots & \ldots & \ldots & \ldots & \ldots & \ldots & \ldots & \ldots \\
k_{268,1} & k_{268,2} & k_{268,3} & \ldots & k_{268,205} & \ldots & \ldots & \ldots & \ldots & k_{268,210} & \ldots & k_{268,268} & k_{268,269} & k_{268,270} \\
k_{269,1} & k_{269,2} & k_{269,3} & \ldots & k_{269,205} & \ldots & \ldots & \ldots & \ldots & k_{269,210} & \ldots & k_{269,268} & k_{269,269} & k_{2691270} \\
k_{270,1} & k_{270,2} & k_{270,3} & \ldots & k_{270,205} & \ldots & \ldots & \ldots & \ldots & k_{270,210} & \ldots & k_{270,268} & k_{270,269} & k_{270,270}
\end{array}\right]\left\{\begin{array}{l}
u_{1} \\
v_{1} \\
w_{69} \\
v_{69} \\
w_{69} \\
u_{70} \\
v_{70} \\
w_{70} \\
\ldots \\
u_{90} \\
v_{90} \\
w_{90}
\end{array}\right\}
$$

Where $k_{i, j}$ is the stiffness coefficient of the global stiffness matrix.

Equating displacements in each direction of all nodes on the barrette by uniform displacements $u_{x}, v_{y}$ and $w_{z}$ and

$$
\left\{\begin{array}{l}
p_{x 1} \\
p_{y 1} \\
p_{z 1} \\
\ldots \\
p_{x 69} \\
p_{y 69} \\
p_{z 69} \\
Q_{x} \\
Q_{y} \\
Q_{z}
\end{array}\right\}=\left[\begin{array}{cccccc}
k_{1,1} & k_{1,2} & \ldots & \ldots & \ldots & k_{1,206} \\
k_{2,1} & k_{2,2} & \ldots & \ldots & \ldots & k_{2,206} \\
k_{3,1} & k_{3,2} & \ldots & \ldots & \ldots & k_{3,206} \\
\ldots & \ldots & \ldots & \ldots & \ldots & \ldots \\
k_{205,1} & k_{205,2} & \ldots & \ldots & \ldots & k_{205,206} \\
k_{206,1} & k_{206,2} & \ldots & \ldots & \ldots & k_{206,206} \\
k_{207,1} & k_{207,2} & \ldots & \ldots & \ldots & k_{207,206} \\
\sum_{i=70} k_{i 1,1} & \sum_{i=70}^{90} k_{i 1,2} & \ldots & \ldots & \ldots & \sum_{i=70}^{90} k_{i 1,206} \\
\sum_{i=70}^{90} k_{i 2,1} & \sum_{i=70}^{90} k_{i 2,2} & \ldots & \ldots & \ldots & \sum_{i=70}^{90} k_{i 2,206} \\
\sum_{i=70}^{90} k_{i 3,1} & \sum_{i=70}^{90} k_{i 3,2} & \ldots & \ldots & \ldots & \sum_{i=70}^{90} k_{i 3,206}
\end{array}\right.
$$

Where $Q_{x}$ is sum of horizontal forces in x-direction on all barrette nodes, $Q_{x}=\sum P_{x}=0 ; Q_{y}$ is sum of horizontal forces in y-direction on all barrette nodes, $Q_{y}=\sum P_{y}=0$; $Q_{z}$ is sum of vertical forces on all barrette nodes, $Q_{z}=\sum P_{z}=P h ; u_{x}$ is uniform displacement in $x$-direction on all barrette nodes, $u_{x=} u_{70}=. .=u_{90} . v_{y}$ is uniform displacement in $y$-direction on all barrette nodes, $v_{x}=v_{70}=$ ..$=v_{90} ; w_{z}$ is uniform displacement in $z$-direction on all barrette nodes, $w_{x=} w_{70}=. .=w_{90} ; i 1=3 i-2, i 2=3 i-1, i 3=3 i$, $j 1=3 j-2, j 2=3 j-1, j 3=3 j$

Solving the above system of linear equations, gives the displacement at each node, in which the vertical displacement is equal to the soil settlement at that node. Substituting barrette displacements from Eq. (19) in Eq. (18), gives contact forces on the barrette.

\section{NUMERICAL RESULTS}

A computer program has been developed for analyzing barrette using CCT using the method outlined in this paper. With the help of this program, an analysis of two verification examples is carried out first to judge the proposed method for both linear and nonlinear analyses. carrying out the summation of rows and columns related to that displacements in Eq. (18), gives the composed coefficients with the force on the barrette $Q_{x}, Q_{y}$ and $Q_{z}$

\begin{tabular}{|c|c|c|c|}
\hline$k_{1,207}$ & $\sum_{i=7}^{90} k_{1, j 1}$ & $\sum_{i=70}^{90} k_{1, j 2}$ & $\sum_{i=70}^{90} k_{1, j 3}$ \\
\hline$k_{2,207}$ & $\sum_{j=70}^{90} k_{2, j 1}$ & $\sum_{j=70}^{90} k_{2, j 2}$ & $\sum_{j=70}^{90} k_{2, j 3}$ \\
\hline$k_{3,207}$ & $\sum_{j=70}^{90} k_{3, j 1}$ & $\sum_{j=70}^{90} k_{3, j 2}$ & $\sum_{j=70}^{90} k_{3, j 3}$ \\
\hline 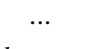 & & & 90 \\
\hline 05,207 & $\sum_{\substack{j=70 \\
90}} k_{205, j 1}$ & $\sum_{\substack{j=70 \\
90}} k_{205, j 2}$ & $\sum_{\substack{j=70 \\
90}} k_{205, j 3}$ \\
\hline 06,207 & $\sum_{j=70}^{90} k_{206, j 1}$ & $\sum_{j=70}^{90} k_{206, j 2}$ & $\sum_{j=70}^{90} k_{206, j 3}$ \\
\hline 07,207 & $\sum_{j=70}^{90} k_{207, j 1}$ & $\sum_{j=70}^{90} k_{207, j 2}$ & $\sum_{j=70}^{90} k_{207, j 3}$ \\
\hline$k_{i 1,207}$ & $\sum_{i=70}^{90} \sum_{j=70}^{90} k_{i 1, j 1}$ & $\sum_{i=70}^{90} \sum_{j=70}^{90} k_{i 1, j 2}$ & $\sum_{i=70}^{90} \sum_{j=70}^{90} k_{i 1, j 3}$ \\
\hline$k_{0} k_{i 2,207}$ & $\sum_{i=70}^{90} \sum_{j=70}^{90} k_{i 2}{ }_{j 1}$ & $\sum_{i=70}^{90} \sum_{j=70}^{90} k_{i 2, j 2}$ & $\sum_{i=70}^{90} \sum_{j=70}^{90} k_{i 2, j 3}$ \\
\hline 3,207 & $\sum_{i=70}^{90} \sum_{j=70}^{90} k_{i 3, j 1}$ & $\sum_{i=70}^{90} \sum_{j=70}^{90} k_{i 3, j 2}$ & $\sum_{i=70}^{90} \sum_{j=70}^{90} k_{i 3, j 3}$ \\
\hline
\end{tabular}
as follows:

Then, a comparative examination of modeling for analyzing single barrette is carried out. Finally case studies for barrettes on the soil of the new area of East Port Said are presented.

\subsection{Validity of Linear Analysis of Single Barrette}

An analytical analysis of a single barrette having a rectangular cross section embedded in a multi-layered soil medium is available in the reference Basu et al.[1]. In the analytical analysis, the differential equations governing the displacements of the barrette-soil system were obtained using variation principles. Closed-form solutions for barrette deflection and axial force along the barrette shaft were then produced by using the method of initial parameters.

The barrette is considered and analyzed for four different cases under different loads, geometries and subsoil conditions. The load on the barrette head and barrette geometry for the chosen cases are listed in Table 1. The subsoil of each case consist of four layers, each layer has a different Modulus of Elasticity $E_{s}$ and Poisson's ratio $v_{s}$ 
as listed in Table 2. The barrette material properties are listed in Table 3.

Table 1 Loads and barrette geometries, Basu et al.[1].

\begin{tabular}{|c|c|c|c|}
\hline Case & Load $[\mathrm{kN}]$ & Height $[\mathrm{m}]$ & Cross section \\
\hline 1 & 3000 & 15 & $0.5[\mathrm{~m}] \times 0.5[\mathrm{~m}]$ \\
\hline 2 & 2500 & 10 & $0.7[\mathrm{~m}] \times 0.7[\mathrm{~m}]$ \\
\hline 3 & 10000 & 40 & $2.8[\mathrm{~m}] \times 0.8[\mathrm{~m}]$ \\
\hline 4 & 8000 & 30 & $2.7[\mathrm{~m}] \times 1.2[\mathrm{~m}]$ \\
\hline
\end{tabular}

Table 2 Subsoil properties, Basu et al.[1].

\begin{tabular}{|c|c|c|c|c|}
\hline Case & Layer No. & $z[\mathrm{~m}]$ & $E_{s}\left[\mathrm{kN} / \mathrm{m}^{2}\right]$ & $v_{s}[-]$ \\
\hline \multirow{4}{*}{1} & 1 & 2 & 10000 & 0.40 \\
\cline { 2 - 5 } & 2 & 5 & 15000 & 0.35 \\
\cline { 2 - 5 } & 3 & 10 & 30000 & 0.30 \\
\hline \multirow{4}{*}{2} & 4 & $\infty$ & 100000 & 0.15 \\
\cline { 2 - 5 } & 1 & 1 & 10000 & 0.40 \\
\cline { 2 - 5 } & 2 & 5 & 15000 & 0.35 \\
\hline \multirow{4}{*}{3} & 3 & 8 & 30000 & 0.30 \\
\cline { 2 - 5 } & 2 & $\infty$ & 80000 & 0.20 \\
\cline { 2 - 5 } & 3 & 3 & 20000 & 0.35 \\
\hline \multirow{4}{*}{4} & 4 & $\infty$ & 25000 & 0.30 \\
\cline { 2 - 5 } & 2 & 2 & 30000 & 0.30 \\
\cline { 2 - 5 } & 3 & 22 & 30000 & 0.20 \\
\hline
\end{tabular}

Where $z$ Layer depth from the ground surface, [m]; $E_{s}$ Modulus of Elasticity, $\left[\mathrm{kN} / \mathrm{m}^{2}\right] ; v_{s}$ Poisson's ratio, [-].

Table 3 Barrette material properties, Basu et al.[1].

\begin{tabular}{l}
$\begin{array}{l}\text { Modulus of Elasticity of the barrette material } E_{c}= \\
2.5 \times 10^{7}\left[\mathrm{kN} / \mathrm{m}^{2}\right]\end{array}$ \\
\hline Poisson's ratio of the barrette material $\quad v_{c}=0.20[-]$ \\
\hline
\end{tabular}

A comparison of results of the single barrette in a multilayered soil medium of the present analysis using flexibility coefficient with those of Basu et al.[1] is presented herein. The height of the barrette is divided into equal elements, and the height of each element is $h$ $=1[\mathrm{~m}]$ in all cases. Both the barrette length and width are divided into four equal elements in each case. In the analysis, barrette material is considered to be elastic and the barrette is analyzed as 1D finite elements.

The barrette settlement $s$ along the barrette height obtained from the present analysis using flexibility coefficient for the four cases of analysis are compared with those of Basu et al.[1] in Figure 7 to Figure 10.

From these results, it can be concluded that the absolute difference between the maximum settlements is ranging between 0.8 [\%] for the first case and 2.0 [\%] for the second case, while the other cases it is only 1.0 [\%]. Also, the absolute differences between the minimum settlements are 7.0 [\%], $4.0[\%], 15.0$ [\%] and 5.0 [\%] respectively.

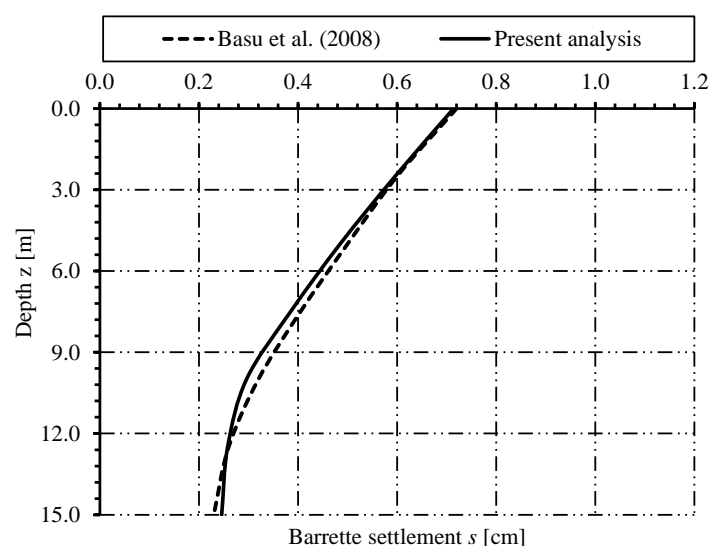

Figure 7 Settlement along the barrette height (case 1).

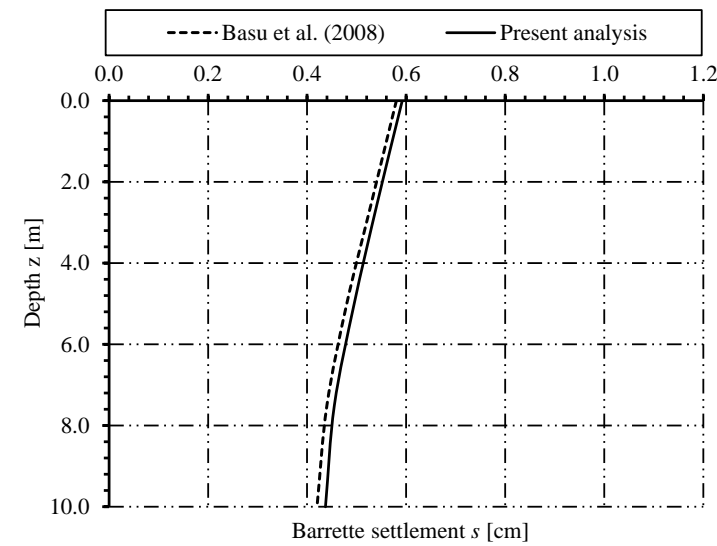

Figure 8 Settlement along the barrette height (case 2).

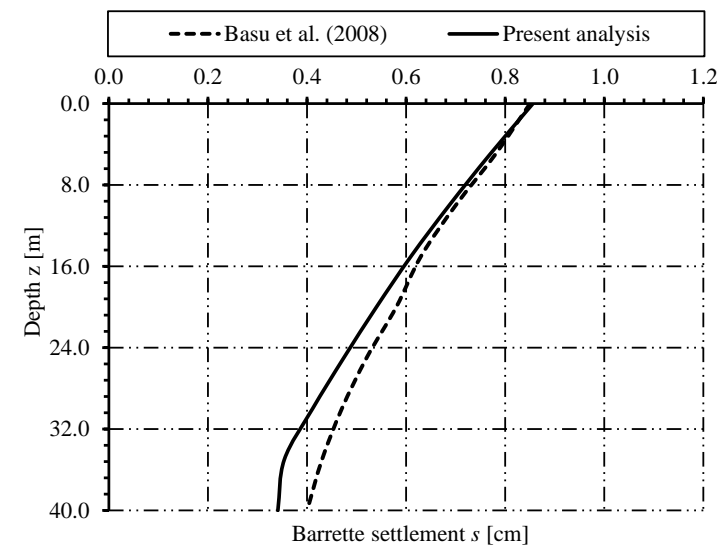

Figure 9 Settlement along the barrette height (case 3).

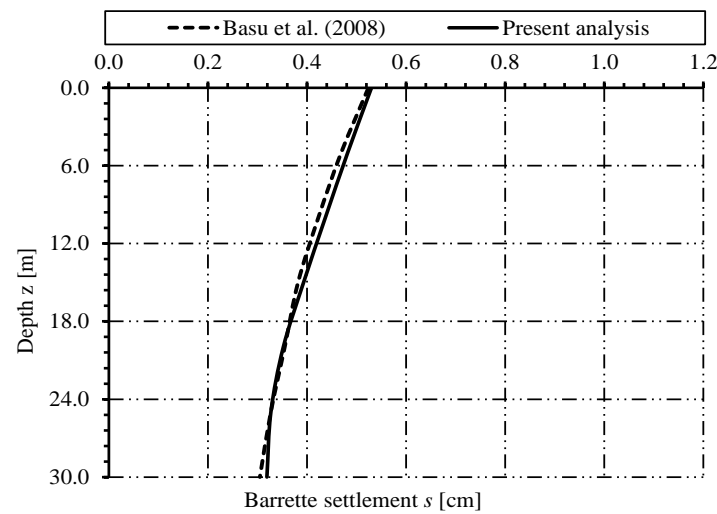

Figure 10 Settlement along the barrette height (case 4).

These results show also that verification results of the present analysis using flexibility coefficient are in good agreement with those of Basu et al.[1]. Results of the 
barrette head settlements are similar to those of Basu et al.[1]. However, regarding results of the base settlements, the difference reached 15.0 [\%] in case of a barrette having a great aspect ratio in the cross section, case (3). The difference in this case is very small when compared to the barrette dimensions, which equals to $0.06[\mathrm{~cm}]$.

\subsection{Validity of Nonlinear Analysis of Single Barrette}

A load tests of a single barrettes having a rectangular cross section embedded in a multi-layered soil medium are available in the reference Thasnsnipan et al. (1998) [29] and Lin et al. (2014) [18]. In the load tests, results of barrette load tests are obtained from Bangkok, Thailand subsoil area and Taipei, Thailand subsoil area respectively.

The load on the barrette head and barrette geometry for the chosen cases are listed in Table 4 and Table 5. The subsoil of each case consists of different layers, each layer having a different Modulus of Elasticity $E_{s}$ and Poisson's ratio $v_{s}$ as listed in Table 6 and Table 7. This soil properties of Thasnsnipan et al. [29]can be found in Plaxis Bulletin [21].

Table 4 Loads and barrette geometries, Thasnsnipan et al. [29].

\begin{tabular}{|c|c|c|}
\hline Load $[\mathrm{kN}]$ & Height $[\mathrm{m}]$ & Cross section \\
\hline 14000 & & \\
\cline { 1 - 1 } 28000 & 61.8 & $0.82[\mathrm{~m}] \times 2.7[\mathrm{~m}]$ \\
\hline 35000 & & \\
\hline
\end{tabular}

Table 5 Barrette geometries, Lin et al. [18].

\begin{tabular}{|c|c|}
\hline Height $[\mathrm{m}]$ & Cross section \\
\hline 44 & $0.80[\mathrm{~m}] \times 2.5[\mathrm{~m}]$ \\
\hline
\end{tabular}

Table 6 Subsoil properties, Plaxis Bulletin [21] and Thasnsnipan et al. [29].

\begin{tabular}{|c|c|c|c|}
\hline Layer No. & $z[\mathrm{~m}]$ & $E_{s}\left[\mathrm{kN} / \mathrm{m}^{2}\right]$ & $v_{s}[-]$ \\
\hline 1 & 12.5 & 5000 & 0.33 \\
\hline 2 & 23 & 60000 & 0.33 \\
\hline 3 & 37 & 80000 & 0.3 \\
\hline 4 & 40 & 20000 & 0.33 \\
\hline 5 & 53 & 80000 & 0.30 \\
\hline 6 & 58 & 20000 & 0.33 \\
\hline 7 & $\infty$ & 80000 & 0.30 \\
\hline
\end{tabular}

Table 7 Subsoil properties, Lin et al. [18].

\begin{tabular}{|c|c|c|c|}
\hline Layer No. & $z[\mathrm{~m}]$ & $E_{s}\left[\mathrm{kN} / \mathrm{m}^{2}\right]$ & $v_{s}[-]$ \\
\hline 1 & 26.46 & 93793 & 0.25 \\
\hline 2 & 28.40 & 253293 & 0.25 \\
\hline 3 & 30.44 & 221593 & 0.25 \\
\hline 4 & 33.60 & 88391 & 0.25 \\
\hline 5 & 36.80 & 131381 & 0.25 \\
\hline 6 & 40.80 & 192106 & 0.25 \\
\hline 7 & 42.44 & 166948 & 0.25 \\
\hline 8 & $\infty$ & 229738 & 0.25 \\
\hline
\end{tabular}

A comparison of the results of a single barrettes in a multi-layered soil medium of the present analysis using flexibility coefficient with those of Thasnsnipan et al.[29] and Lin et al. [18] are presented herein, The height of the barrette is divided into equal elements, each element has a height of $h=1.0[\mathrm{~m}]$. Both the barrette length and width are divided into four equal elements. The barrettes are analyzed nonlinearly using a hyperbolic function to represent the real load settlement curve relation. In the analysis, the barrette is assumed to be fully rigid having a uniform settlement.

A limit barrette load $Q l$ has been used as a parameter geometry for the hyperbolic curve of nonlinear response of load settlement relation. Russo [26] suggested a limiting shaft friction not less than $180\left[\mathrm{kN} / \mathrm{m}^{2}\right]$ meeting undrained shear strength of $200\left[\mathrm{kN} / \mathrm{m}^{2}\right]$. To carry out the present nonlinear analysis a limit shaft friction of $q l$ $=220\left[\mathrm{kN} / \mathrm{m}^{2}\right]$ is considered, which gives a limit barrette load of $Q l=96[\mathrm{MN}]$ to compare with the result with those of Thasnsnipan et al. [29], where it is calculated from:

$$
Q l=q l \times(W+L) \times 2 \times H
$$

Where $W$ barrette width, $[\mathrm{m}] ; L$ barrette length, $[\mathrm{m}] ; H$ barrette height, [m].

A limit barrette load of $Q l=50[\mathrm{MN}]$ is taken, to compare with the result with those of Lin et al.[18], where it is assumed from the load settlement curve of Lin et al. [18].

The barrette load-settlement relations obtained from the present nonlinear analysis using flexibility coefficient are compared with those of the load tests carried out by Thasnsnipan et al.[29] and Lin et al. [18] in Figure 11 and Figure 12 respectively. From these figures, it can be concluded that the difference of the measured and computed settlement is less than 10.0 [\%], which have a very small value of $0.04[\mathrm{~cm}]$ and $0.16[\mathrm{~cm}]$ compared with those of Thasnsnipan et al.[29] and Lin et al. [18] respectively. It is also very small when compared to the barrette dimensions. It also shows that the verification of the load-settlement behavior of the present nonlinear analysis are in good agreement with those of measured load settlement tests carried out by Thasnsnipan et al.[29] and Lin et al. [18].

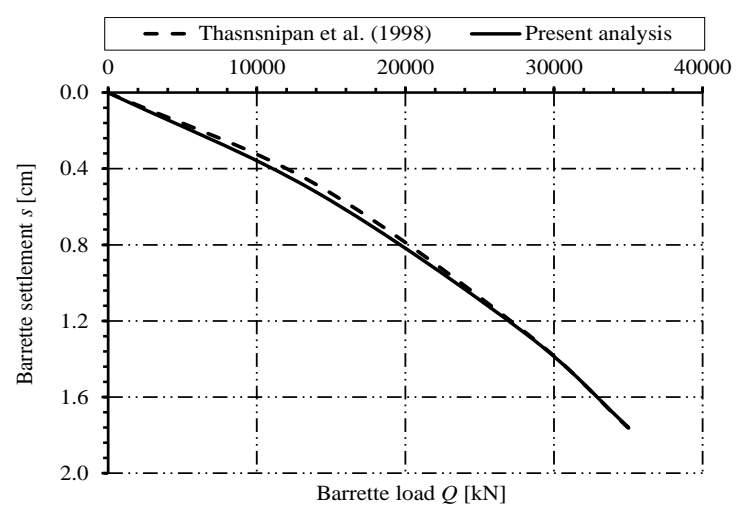

Figure 11 Load settlement curve, Thasnsnipan et al. [29]. 


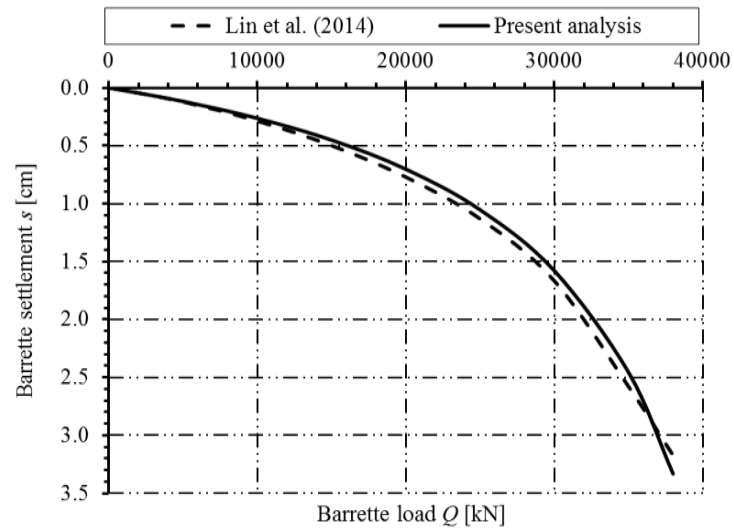

Figure 12 Load settlement curve, Lin et al. [18].

\subsection{Comparative Examinations of Modeling for Analyzing Single Barrette}

A single barrette having a rectangular cross section embedded in different subsoil conditions is analyzed using the two different models based on $C C T$ technique:

1. 3D finite element model.

2. Flexibility coefficient model.

Results of 3D finite element model are compared with those using flexibility coefficient model. In 3D finite element model, the barrette-soil system are represented by block elements, each consists of eight nodes. The composed coefficient technique $C C T$ is implemented in both 3D finite element and flexibility coefficient models. In this case, the barrette is treated as a rigid body having uniform settlement. This technique reduces the commotional time and the size of the problem as these two terms considered as main difficulties in the three dimensional problems.

The purpose of the comparative study is showing the limitations and differences in both results and also to be a guideline to determine which model may be preferably used in the analysis. The barrette shown in Figure 13 is considered and analyzed linearly for twelve different cases of loads, subsoil and geometries. Analysis covered a wide range of variables of barrette length $L$ and barrette height $H$. The effect of these variables on the settlement is also investigated. The barrette geometry for the chosen cases is listed in Table 8, while the loads on the barrette head of each case are listed in Table 9.

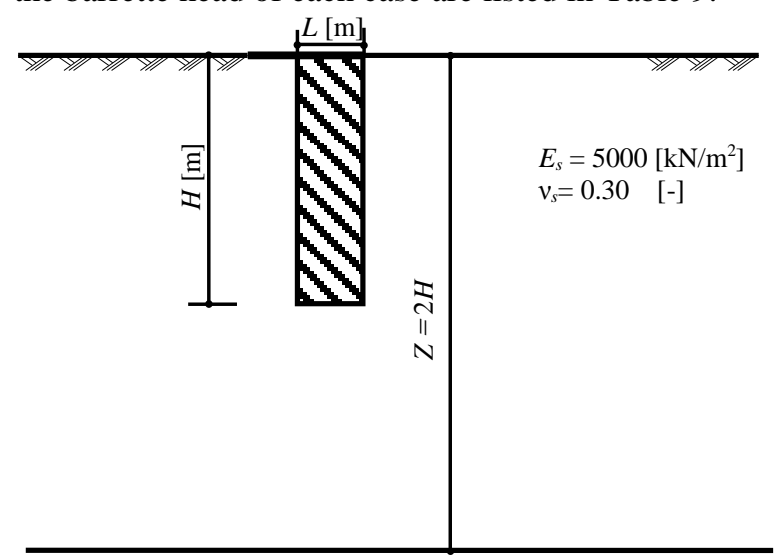

Figure 13 Single barrette with subsoil (A).
Table 8 Barrette geometries.

\begin{tabular}{|c|c|c|c|c|}
\hline Length/Height & $\mathrm{L}=1.5$ & $\mathrm{~L}=2.0$ & $\mathrm{~L}=2.5$ & $\mathrm{~L}=3.0$ \\
\hline $\mathrm{H}=10$ & Case 1 & Case 2 & Case 3 & Case 4 \\
\hline $\mathrm{H}=15$ & Case 5 & Case 6 & Case 7 & Case 8 \\
\hline $\mathrm{H}=20$ & Case 9 & Case 10 & Case 11 & Case 12 \\
\hline
\end{tabular}

Table 9 Barrette loads.

\begin{tabular}{|c|c|c|c|c|}
\hline Length/Height & $L=1.5$ & $L=2.0$ & $L=2.5$ & $L=3.0$ \\
\hline$H=10$ & 750 & 1000 & 1250 & 1500 \\
\hline$H=15$ & 900 & 1200 & 1500 & 1800 \\
\hline$H=20$ & 1050 & 1400 & 1750 & 2100 \\
\hline
\end{tabular}

Table 10 Subsoil properties.

\begin{tabular}{|c|c|c|c|}
\hline Soil & $z[\mathrm{~m}]$ & $E_{s}\left[\mathrm{kN} / \mathrm{m}^{2}\right]$ & $v_{s}[-]$ \\
\hline (A) & $2 H$ & 5000 & 0.30 \\
\hline & $0.4 H$ & 5000 & 0.40 \\
\cline { 2 - 4 } (B) & $1.2 \mathrm{H}$ & 8000 & 0.35 \\
\cline { 2 - 4 } & $2 \mathrm{H}$ & 10000 & 0.30 \\
\hline \multirow{2}{*}{ (C) } & $0.6 \mathrm{H}$ & 5000 & 0.40 \\
\cline { 2 - 4 } & $2 \mathrm{H}$ & 25000 & 0.30 \\
\hline \multirow{2}{*}{ (D) } & $0.6 \mathrm{H}$ & 25000 & 0.30 \\
\cline { 2 - 4 } & $2 \mathrm{H}$ & 5000 & 0.40 \\
\hline
\end{tabular}

The twelve cases of loads and geometries are analyzed with different subsoil's as listed in Table 10 as follows:

- Soil (A) Single layer.

- Soil (B) Three different layers extended from a weak layer to a hard one.

- Soil (C) Two different layers, the first is a weak layer and the second is a hard one.

- Soil (D) Two different layers, the first is a hard layer and the second is a weak one.

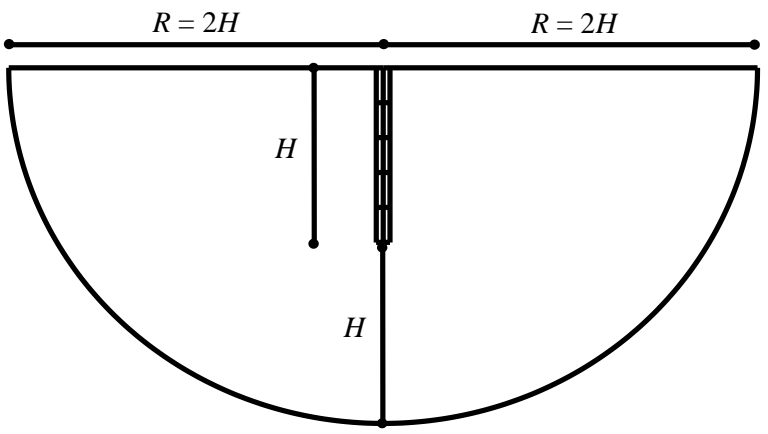

a) Theoretical isotropic half-space medium

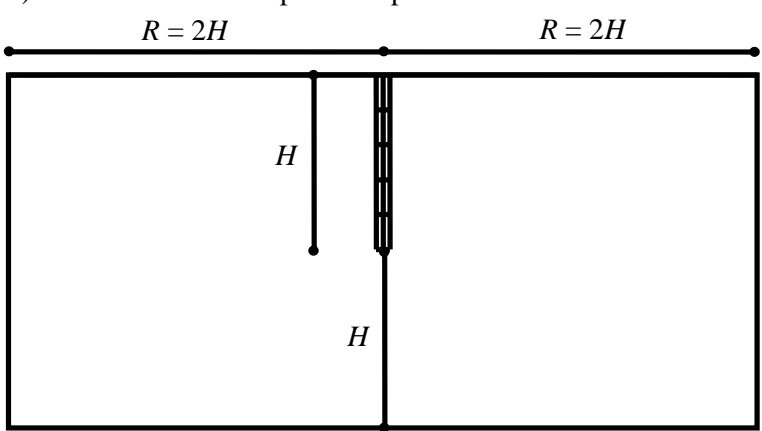

b) Considered soil medium in the analysis

Figure 14 Soil medium.

A single barrette is analyzed in a single soil layer, and the height of the barrette is divided into equal elements, of $1.0[\mathrm{~m}]$ height each. The barrette length and width are 
divided into two equal elements. To ensure full interaction between the isotropic elastic half-space soil medium and the barrette, the dimension of the soil around the barrette is extended enough, as shown in Figure 14. The barrette is analyzed linearly and the barrette is assumed to be fully rigid having a uniform settlement.

In the 3D finite element model, the barrette and the soil elements are solved as double symmetric system to reduce the number of equations to quarter. Consequently, the computational time is also reduced.

The barrette settlements obtained from both analysis are compared. Figure 15 to Figure 18 show the settlement results and the difference in the calculated settlements for the two models.

From these figures, it can be concluded that:

- For a single soil layer, Soil (A), settlements are identical for both models. The maximum difference in the settlement of both models lies between $0.1[\mathrm{~cm}]$ and $0.2[\mathrm{~cm}]$, which is equal to a very small value when compared with the barrette dimensions.

- For soil consists of three different layers extended from a weak layer to a hard layer, Soil (B), settlements are identical for both models. The maximum difference in the settlement between both models is 0.1 $[\mathrm{cm}]$, which is very small when compared with the barrette dimension.

- For two different layers where the first is a weak layer and the second is a hard layer, Soil (C), settlements are identical for both models. The maximum difference in the settlement between both models lies between $0.1[\mathrm{~cm}]$ and $0.2[\mathrm{~cm}]$, which is very small when compared with the barrette dimensions.

- For two different layers where the first is a hard layer and the second is a weak one, Soil (D), the maximum difference in settlements between both models is high and maybe twice. It is found that settlements from 3D finite element model are less than those of flexibility coefficient model. This is related to, in 3D finite element mode, the first harder layer is to be a support for the next weaker soil layer, where the soil is treated as continuum structure connected together and maybe resist soil tension. In this case interface elements between the two layers could be inserted to enhance the flexibility coefficient technique.

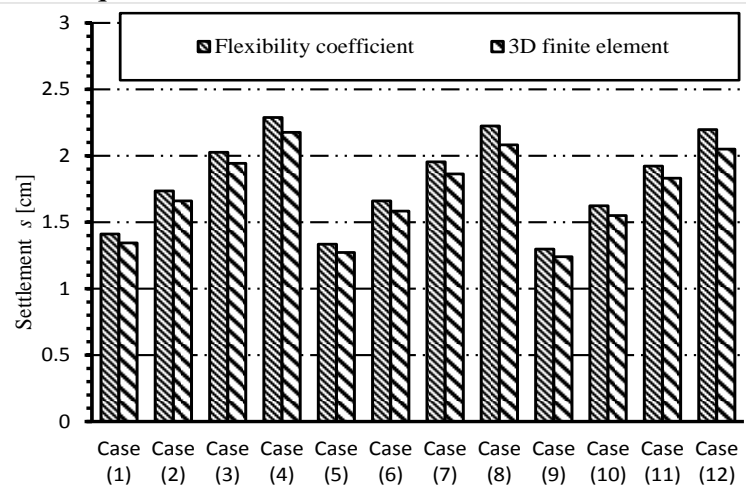

Figure 15 Comparison between settlements, Soil (A).

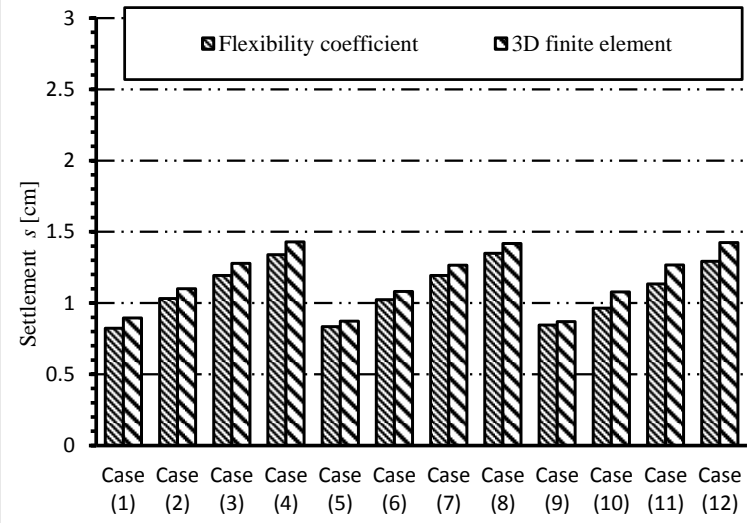

Figure 16 Comparison between settlements, Soil (B).

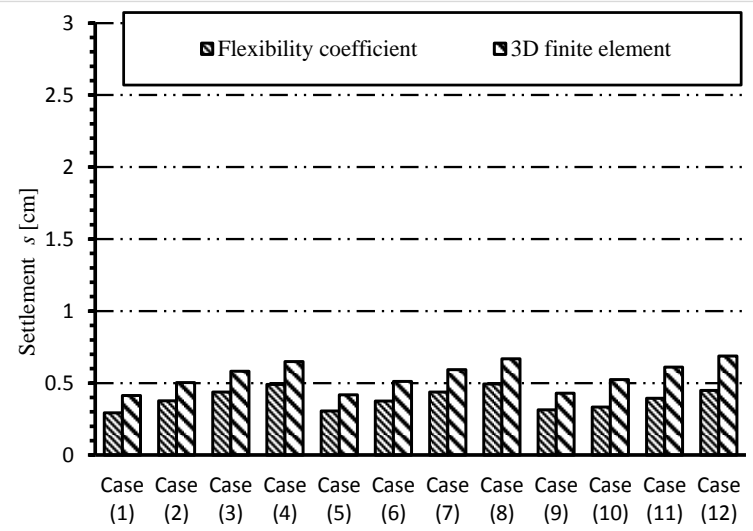

Figure 17 Comparison between settlements, Soil (C).

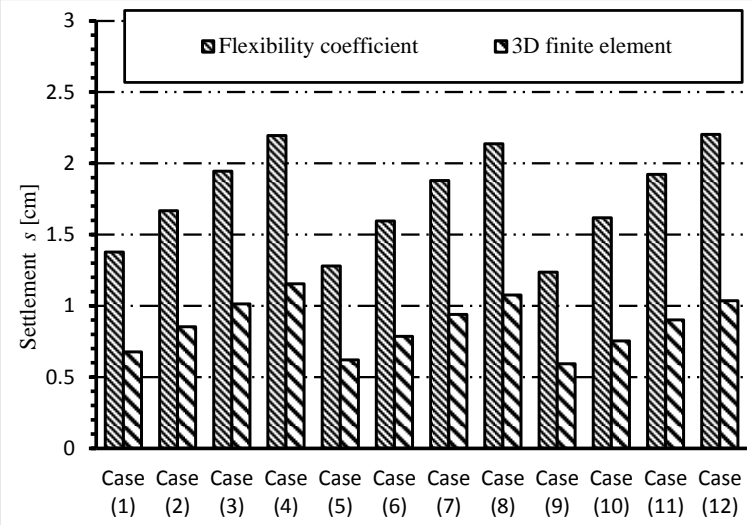

Figure 18 Comparison between settlements, Soil (D)

\subsection{Case Studies of a Single Barrette}

This section presents the main features of the numerical models used in analyzing the behavior of single barrette in a real subsoil. The subsoil of East Port Said area is considered as the proposed real subsoil in these case studies. The reason is that the existing heavy loaded structures in East Port Said suffered from settlement problems due to the presence of extended soft clay layers. The typical subsoil layers of East Port Said area, as presented by Hamza, M.[9] in Table 13, is considered in the analysis. The different case studies under investigation are also described. Every case is examined in a parametric study. The study covered different barrette lengths $L$ with different barrette heights $H$ for a constant barrette width $W$ of 1.0 [m]. The effect of these variables on the barrette loads at certain settlement is 
also investigated. Furthermore, the analysis is carried out considering various calculation methods. The main features of the most effective numerical methods suitable for the single barrette analysis in East Port Said clay are also discussed. The main variables of the parametric study are described in the next paragraphs.

\subsubsection{Barrette Properties}

Twelve case studies of single barrettes are considered as given in Table 11.

Table 11 Studied cases of a single barrette.

\begin{tabular}{|c|c|c|c|c|}
\hline Length/Height & $L=1.5$ & $L=2.0$ & $L=2.5$ & $L=3.0$ \\
\hline$H=24$ & Case 1 & Case 2 & Case 3 & Case 4 \\
\hline$H=30$ & Case 5 & Case 6 & Case 7 & Case 8 \\
\hline$H=36$ & Case 9 & Case 10 & Case 11 & Case 12 \\
\hline
\end{tabular}

The subsoil of each case assumed to be the typical soil properties of East Port-Said area as given in Table 13, each layer has a different Modulus of Elasticity $E_{s}$ and Poisson's ratio $v_{s}$. The barrette material properties are listed in Table 12.

Table 12 Barrette material properties.

Modulus of Elasticity of the barrette material $E_{c}=$ $2.5 \times 10^{7}\left[\mathrm{kN} / \mathrm{m}^{2}\right]$

Poisson's ratio of the barrette material $\quad v_{c}=0.20[-]$

Table 13 Subsoil properties, Hamza et al. [9].

\begin{tabular}{|c|c|c|c|}
\hline Layer No. & $z[\mathrm{~m}]$ & $E_{s}\left[\mathrm{kN} / \mathrm{m}^{2}\right]$ & $v_{s}[-]$ \\
\hline 1 & 5 & 2400 & 0.2 \\
\hline 2 & 13.5 & 30000 & 0.25 \\
\hline 3 & 28.5 & 8120 & 0.2 \\
\hline 4 & 38.5 & 9940 & 0.2 \\
\hline 5 & 48.5 & 11340 & 0.2 \\
\hline 6 & 58.5 & 12810 & 0.2 \\
\hline 7 & 92.5 & 60000 & 0.2 \\
\hline 8 & 120 & 144000 & 0.2 \\
\hline
\end{tabular}

\subsubsection{Numerical Analysis}

In this paper, comparative tests of numerical models for analyzing single barrette in East Port Said deep clay layers are performed. For the purpose of comparative investigations, two different models of single barrette are considered in a total of 48-case studies. The analysis is carried out by the following methods:

1. Elastic barrette in a continuum soil medium.

2. Rigid barrette in a continuum soil medium.

The load-settlement relation is determined according to:

a) Nonlinear analysis of a single barrette using hyperbolic function.

b) Linear analysis of a single barrette.

The availability of the above mentioned analysis methods and load-settlement models provides the researcher with a wide variety of numerical models that can handle the problem of single barrette as indicated in Table 11. In this analysis, many case studies of single barrette are analyzed using different numerical models in order to explore the effect of the type of calculation method on the results.

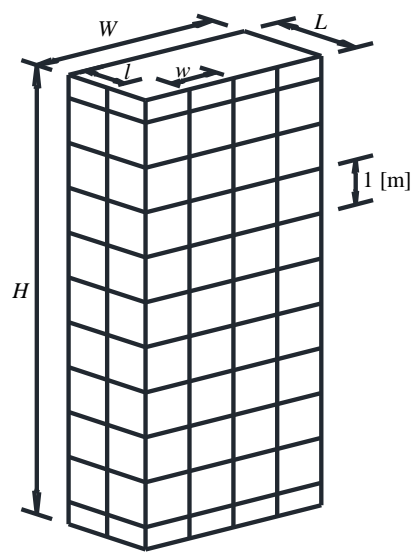

Figure 19 Surface element of the single barrette.

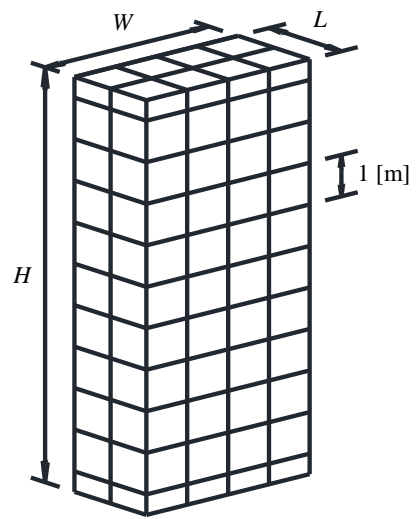

Figure 20 Barrette representing by 3D finite elements.

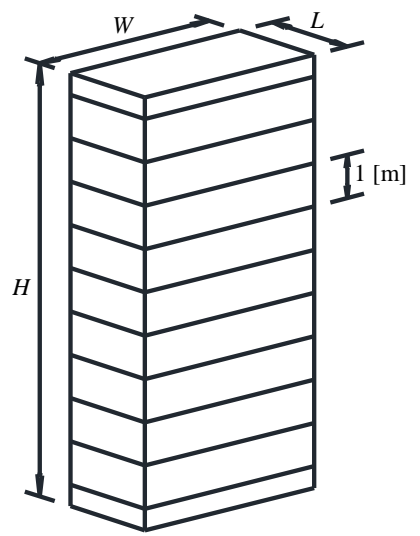

Figure 21 Barrette representing by 1D finite elements.

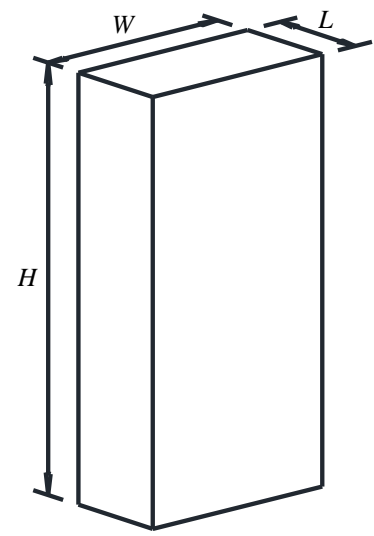

Figure 22 Barrette representing as rigid elements.

Twelve case studies are presented with variables including; the height, length and width of the barrette 
which are divided into equal elements, and the height of each element is $h=1.0[\mathrm{~m}]$, in all cases. Both the barrette length and width were divided into equal elements, the length and the width of each element is $l=$ $w=0.5[\mathrm{~m}]$, in all cases as shown in Figure 19 to Figure 22.

\subsubsection{Limit Barrette Load}

A limit barrette load $Q l[\mathrm{kN}]$ has been used as parameter geometry for the hyperbolic curve of nonlinear response of load settlement relation. Russo suggested limit shaft friction not less than $q l=180 \mathrm{kN} / \mathrm{m}^{2}$ meeting undrained shear strength of $200\left[\mathrm{kN} / \mathrm{m}^{2}\right]$. To carry out the present analysis a limit shaft friction of $q l=180 \mathrm{kN} / \mathrm{m}^{2}$ has been assumed, the limit barrette load for barrettes of different dimensions has been calculated from Eq (20). Using this equation, limit barrette load considered in the analysis for barrette dimensions which are presented in Table 14 .

Table 14 Limit barrette load $Q_{l}[\mathrm{kN}]$ for different barrette geometries.

\begin{tabular}{|c|c|c|c|c|}
\hline Length/Height & $L=1.5$ & $L=2.0$ & $L=2.5$ & $L=3.0$ \\
\hline$H=24$ & 21600 & 25920 & 30240 & 34560 \\
\hline$H=30$ & 27000 & 32400 & 37800 & 43200 \\
\hline$H=36$ & 32400 & 38880 & 45360 & 51840 \\
\hline
\end{tabular}

\subsubsection{Guideline of Barrette Stiffness}

One of the difficulties that arise when analyzing a three dimensional problem, such as barrette in a continuum soil medium, is the huge number of $3 \mathrm{D}$ finite elements required for the analysis. Consequently, a long computational time is needed. Before performing the analysis routine, an examination for the used element type and barrette rigidity is carried out. This examination depends on that barrette itself as a great block of concrete which may be considered as rigid enough in the long direction. This property advantage maybe used to simplify the problem and to accelerate the analysis.

\subsubsection{Barrette Elasticity}

To analyze the barrette as an elastic material, two different methods are used in this paper. The first depends on 3D finite elements representing the barrette by its natural geometry, Figure 20 . The second method using 1D finite elements in the $\mathrm{z}$-direction representing the barrette as line elements in the direction of its height, Figure 21. The twelve cases listed before are analyzed using the two different types of elements, and the results of reactions, settlements and elapsed time are compared, as shown in Figure 23 to Figure 26.

\subsubsection{Conclusion of Barrette Elasticity}

From Figure 23 to Figure 26 it can be concluded that:

- The absolute difference in the base reactions using 1D and 3D finite elements is less than 1.18 [\%], while that for shaft reactions is less than 0.08 [\%].

- The elapsed time to analyze the single barrette will be decreased by about 85 [\%] when using 1D finite elements.

- The difference in the settlement when using 1D and 3D finite elements are less than 0.25 [\%].

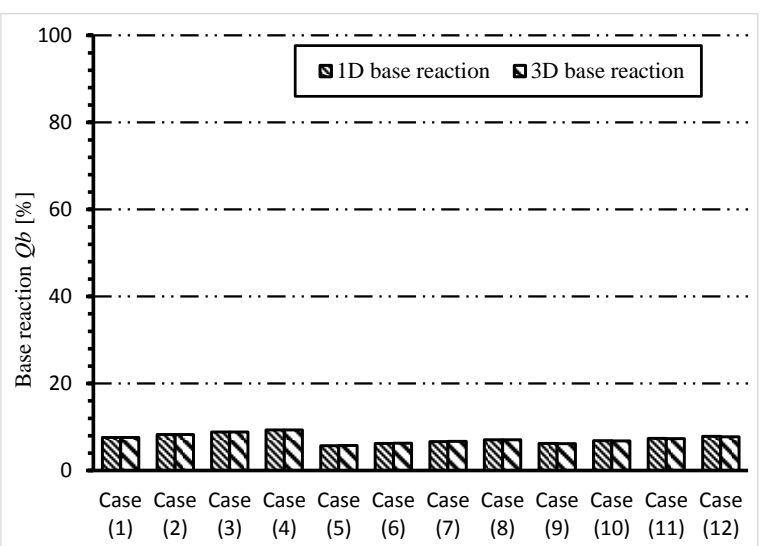

Figure 23 Base reaction using 1D and 3D finite elements.

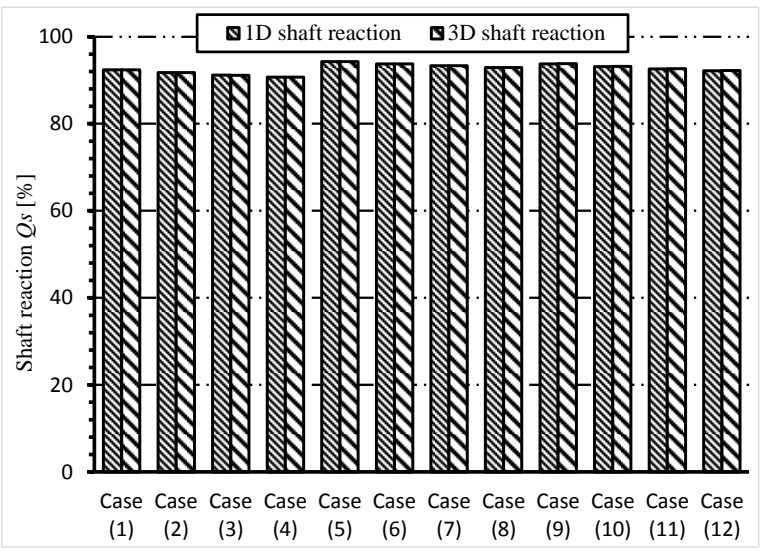

Figure 24 Shaft reaction using 1D and 3D finite elements.

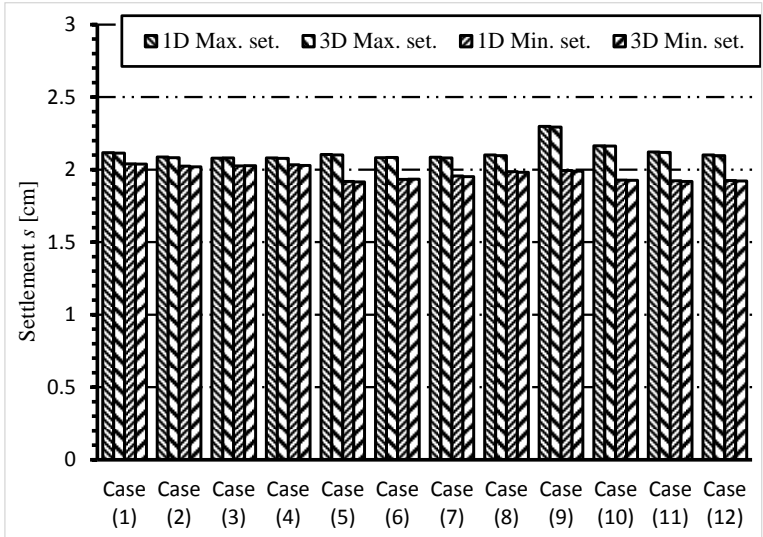

Figure 25 Max. and Min. settlement when using 1D and $3 \mathrm{D}$ finite elements.

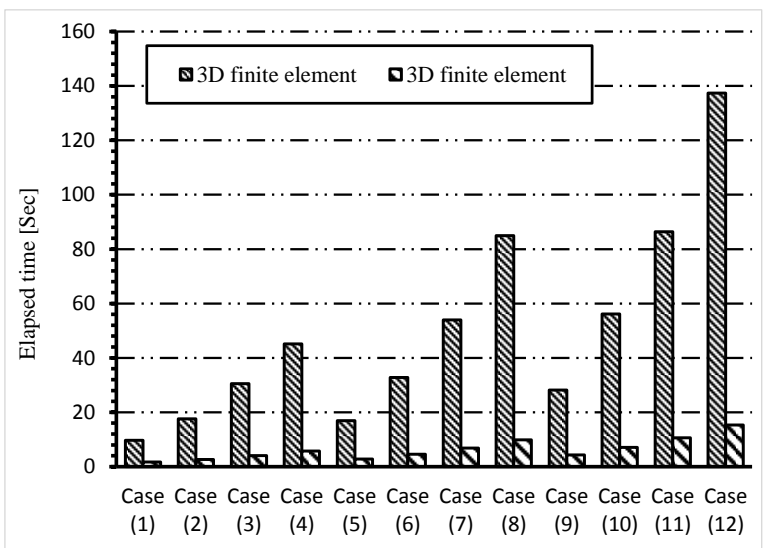

Figure 26 Elapsed time [sec] when using 1D and 3D finite elements. 


\subsubsection{Barrette Rigidity}

Settlement along the barrette height is considered the main important value in all barrette results. Therefore, in this section an examination is carried out for considering the barrette as one unit having a uniform settlement along its height or as an elastic body having a nonuniform settlement along its height. In the first assumption the barrette is treated as a full rigid body which obeys the rigid body movement, while in the second the barrette is treated as an elastic body taking into account the elastic property of its material.

The twelve cases listed before for single barrette are analyzed as a full rigid barrette in a continuum soil medium, Figure 22, and as an elastic barrette in a continuum soil medium, Figure 21. Results of the settlements are compared using both linear and nonlinear analyses, as shown in Figure 27, Figure 28.

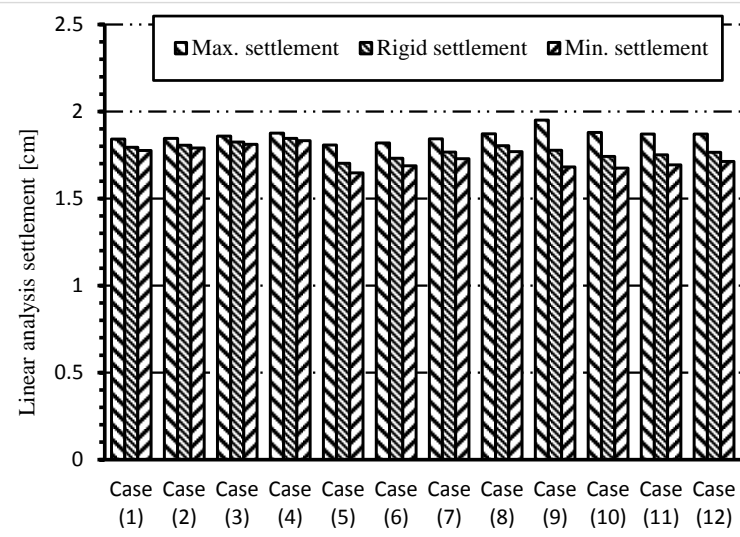

Figure 27 Maximum, minimum and rigid settlement using linear analysis.

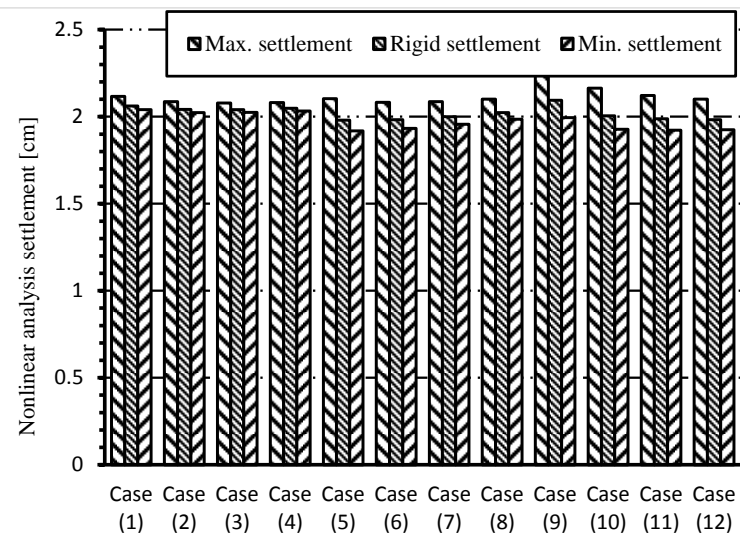

Figure 28 Maximum, minimum and rigid settlement using nonlinear analysis.

\subsubsection{Conclusion of Barrette Rigidity}

From Figure 27, Figure 28, it can be concluded that:

- The absolute difference between the maximum settlement considering a rigid barrette and an elastic barrette for both linear and nonlinear analyses is about 9.74 [\%]. It occurred in case (9), and is less than 8 [\%] in all the remaining other cases.

- The absolute difference between the minimum settlement considering a rigid barrette and an elastic barrette for both linear and nonlinear analyses is about $4.78[\%]$. It is occurred in case (9), and is less than 4 $[\%]$ in the other cases.
- The maximum difference occurs in barrettes having a long height in the soil.

- Barrettes of small cross sections gave higher settlement difference.

- In spite of the relatively large differences between the maximum settlements which ranged between 9.74 [\%] and 4.78 [\%], their actual value are very small, $0.204[\mathrm{~cm}]$ and $0.1[\mathrm{~cm}]$, respectively.

\section{CONCLUSIONS}

An application of $C C T$ on barrettes as large-section supports is presented. The proposed technique considers the $3 \mathrm{D}$ full interactions between barrette and soil. From application of $C C T$ technique on real soil, it can be concluded that:

- Both flexibility coefficient and 3D finite element models can be used safely in the linear analysis of single barrette in cases of half space soil and soil consists of different layers extended from weak to hard layers and the results are identical.

- For soils that consist of different layers extended from hard layer to weak one, the maximum difference in the settlement between both models is high and reach twice. It is found that settlements from $3 \mathrm{D}$ finite element model are less than those of flexibility coefficient model. This is related to, in 3D finite element mode, the first harder layer is to act as a support for the next weaker soil layer, where the soil is treated as continuum structure connected together and maybe resist soil tension. In this case interface elements between the two layers maybe inserted to enhance the results.

- Flexibility coefficient model can be used safely to model all cases of soil conditions.

- Due to the less number of nodes in flexibility coefficient model rather than 3D finite element model, the first model consumes less computation time in the analysis.

- Treating the barrette as an elastic body and representing the barrette by either $1 \mathrm{D}$ or $3 \mathrm{D}$ finite elements, gives nearly the same results. This conclusion is used in this paper, when analyzing the barrette as an elastic body.

- Treating the barrette as a rigid body due to its high rigidity in the direction of its height, gives nearly the same results as treating it as an elastic body.

\section{REFERENCES}

[1] Basu, D., Prezzi, M., Salgado, R., Chakraborty, T. (2008): Settlement analysis of piles with rectangular cross sections in multi-layered soils.

[2] Chandrupatla, T., Belegundu, A. (2000): Introduction to Finite Elements in Engineering Prentice/Hall of India, New Delhi.

[3] Chieruzzi, G., Roy, D., Ayoubian, A. (2013): Measured and Modeled Settlement of Power Plant on Piled Raft Foundation. DIF, 37 $7^{\text {th }}$ Annual Deep Foundations Conference, Houston, TX. 
[4] El Gendy, M. (2007): Formulation of a composed coefficient technique for analyzing large piled raft, Scientific Bullettin, Faculty of Engineering, Ain Shams University, Cairo, Egypt. Vol. 42, No. 1, March, pp. 29-56.

[5] El Gendy, M., El Araby, I., Kamal, M. (2013): Comparative Examination of Single Bored Piles Using International Codes, Scientific Research Journal of Engineering, No. 5, 2013 pp: 796806.

[6] El Gendy, M., El Araby, I., Kamal, M. (2014): Comparative analysis of large diameter bored piles using international codes, Deep Foundations Institute Journal, Engineering, Volume 8, No. 1, 2014 pp: 15-26.

[7] El Labban, A. (2011): Comparative Studies for Piled Raft Resting on Port Said Clay, M.Sc. Thesis, Port Said University, 2011.

[8] Fellenius, B., Altaee, A., Kulesza, R., Hayes, J. (1999): O-Cell testing and FE analysis of 28-mdeep barrette in Manila, Philippines, Journal of Geotechnical and Environmental Engineering, Vol. 125, No. 7, 1999, pp. 566-575.

[9] Hamza, M., Hamed, H. (2000): Three Dimensional Soil-Structure Analysis of PortSaid East Quay Wall, Maritime Engineering and Ports II, C.A. Brebbia \& J. Olivella, ISBN $1-85312-829-5$

[10] Hattab,F.(2007): Vergleichende Untersuchungen numerischer Modelle für die Berechnung von Pfahlplattengründungen, Diplomarbeit, Matr.-Nr.:537502, NIVERSITÄT SIEGEN.

[11] Ibrahim, F., El Gendy, M., Salib, R., El Kamash, W. (2009): Nonlinear analysis of piled raft with 3D-space structure, Port-Said Engineering Research Journal, V. 13, no. 2, Sep. 2009, Egypt.

[12] Kamash, W. (2009): Analysis of 3D-Structures Resting on Piled Raft, P.HD. Theses, Suez Canal University, Faculty of Engineering 2009.

[13] Kamash, W. (2009): Analysis of Structures on Piled Raft under Earthquake Excitations, Ain Shams Journal of Civil Engineering, Vol. 1.No.1, March 2009, pp. 203-2141

[14] Kamash, W. (2012): The positioning and thickness effect for soft clay layer on 3Dbuilding resting on piled raft, Ain Shams Engineering Journal (2012) 3, 17-26

[15] Kamash, W. (2013): Studying the effect of soft clay layer with different thicknesses on piled raft considering the superstructure, CSCE 2013 General Conference-Congrès général 2013 de la SCGC, Canadian society of Civil Engineering, At Montreal, Québec , Canada, May 2013

[16] Kamash, W., El Gendy, M., Salib, R., Kandil, M. (2014): Studying of Shear Walls with Piled Raft over Soft Soil against Seismic Loads, Port Said Engineering Research Journal, Faculty of Engineering-Port Said University, Volume 18, No. 1, March 2014 pp: 144-152.
[17] Lei, G., Hong, X., Shi, J. (2007): Approximate three-dimensional analysis of rectangular barrette-soil-cap interaction, Can. Geotechnical Journal, Volume 44, 2007 pp: 781-796

[18] Lin, S., Lu, F., Kuo, C., Su, T., Mulowayi, E. (2014): Axial capacity of barrette piles embedded in gravel layer, Journal of GeoEngineering, Vol. 9, No. 3, December 2014, pp. 103-107.

[19] Mobarak, W. (2010): Effect of tie girders on pile caps in Port Said. M.Sc. Thesis, Faculty of Engineering, Suez Canal University, Port Said.

[20] Moubarak, A. (2013): Analysis of Flexible Retaining Walls and Piled Rafts as Settlement Reducer in Port-Said, PhD thesis, Port Said university, 2013.

[21] Plaxis Bulletin, Issue 32/ autumn 2012, Delft, The Netherlands.

[22] Rabiei, M. (2009): Parametric Study for Piled Raft Foundations, EJGE, Vol. 14, Bund. A.

[23] Rabiei, M. (2010): Effect of Pile Configuration and Load Type on Piled Raft Foundations Performance, Deep Foundations and Geotechnical Institute Testing (ASCE-GSP 205), Section 1: Piled Raft System and SoilStructure Interaction, pp. 34-41

[24] Rabiei, M. (2016): Piled Raft Design Strategies for High Rise Buildings, Geotechnical and Geological Engineering, February 2016, Volume 34, Issue 1, pp 75-85

[25] Reda A. (2009): Optimization of reinforced concrete piled raft. M.Sc. Thesis, Faculty of Engineering, Suez Canal University, Port Said.

[26] Russo, G. (1998): Numerical analysis of piled rafts, Int. J. Numer. Anal. Meth. Geomech., 22, 477-493

[27] Seo, H., Basu, D., Prezzi, M., Salgado, R. (2009): Load-settlement response of rectangular and circular piles in multilayered soil, Geotechnical and Geoenvironmental Eng., March 2009, pp 420-430

[28] Shulyatiev, O., Dzagov, A., Bokov, I., Shuliatev $S$. (2013): Correction of soil design parameters for the calculation of the foundation based on the result of barrettes static load test, $18^{\text {th }}$ International Conference on Soil Mechanics and Geotechnical Engineering, Paris 2013

[29] Thasnanipan, N., Maung, A. and Tanseng, P. (1998): Barrettes Founded in Bangkok Subsoils, Construction and Performance. Proceedings of the Thirteenth Southeast Asian Geotechnical Conference, November 16-20 1998, Taipei, ROC.

[30] Thasnanipan, N., Maung, A. and Aye, Z. (2001): Record load test on a large barrette and its performance in the layered soils of Bangkok, $5^{\text {th }}$ international conference on deep foundation practice incorporating Piletalk, 4-6 April 16-20 2001, Singapore. 